
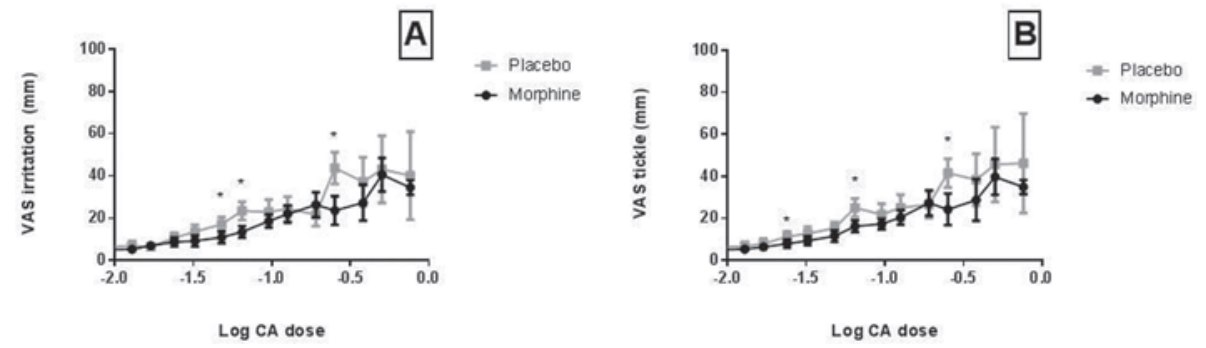

Abstract P104 Figure 1 Figure showing the effects of morphine and placebo on noxious sensations (A "Irritation" B "tickle") evoked by increasing doses of citric acid in our cough challenge ${ }^{*} p<0.05$.

Results Compared with placebo, low dose morphine significantly reduced the VAS scores for tickle and irritation during the citric acid challenge $(p=0.021, p=0.039)$, however UTC, taste and the number of coughs evoked were not improved $(p=0.105, p=0.167$, and $p=0.337)$. Of particular note, morphine had no significant impact on $\mathrm{C} 2$ compared with placebo $(\mathrm{p}=0.611)$.

Conclusions This data shows that treatment with low dose morphine significantly reduces the noxious sensations driving cough. The effects on tickle and irritation appear more important than any impact on the UTC, numbers of coughs triggered or the traditional $\mathrm{C} 2$ endpoint, suggesting that reducing somatic sensations may be an important component of the mode of action of opioids in the treatment of cough.

\section{P105 DOES FENO PREDICT CLINICAL CHARACTERISTICS IN CHRONIC COUGH?}

M Haji Sadeghi, C Wright, S Hart, M Crooks, A Morice. Hull York Medical School, Hull, UK

\subsection{6/thoraxjnl-2017-210983.247}

Objectives In this study we aimed to explore the efficacy of FeNO (The fraction of exhaled nitric oxide) measurement in determining airway inflammatory phenotype in chronic cough patients. FeNO is a biomarker of eosinophilic inflammation. Sequential patients attending a specialist cough clinic were classified as low FeNO (FeNO $\leq 20 \mathrm{ppb}$ ) or high FeNO (FeNO $\geq 30 \mathrm{ppb}$ ) to evaluate the profile of other eosinophilic biomarkers (blood and sputum), cough frequency, and demographics to determine if they exhibited phenotypic variability.

Methods In total 49 patients completed the baseline visit. Correlations between FeNO, blood and sputum eosinophil cell count were assessed. We then compared the objective and subjective measurements of cough in patients with high FeNO and low FeNO at baseline. 24 hour cough counts were measured using the Hull Automated Cough Counter (HACC). Hull Airways Reflux Questionnaire (HARQ) and Leicester Cough Questionnaire (LCQ) were applied to measure cough subjectively.

Results There was a marked gender difference between groups with the low FeNO group having 90\% women whereas the sexes were equally represented in the high FeNO cohort. The predominantly female, low FeNO group had more than twice the number of coughs recorded. Again at baseline there was a significant difference in HARQ and LCQ scores between high and low FeNO groups. Patients with low FeNO suffered more from cough symptoms in comparison with patients with high FeNO according to the 24 hour cough count, HARQ and LCQ scores. FeNO value had a strong correlation with blood and sputum eosinophil count $(\mathrm{r}=0.79$ and $\mathrm{r}=0.65 \mathrm{p}<0.001$ respectively).

Conclusions By evaluating the demographic data, 24 hours cough count, HARQ and LCQ in high FeNO and low FeNO groups, different characteristics between these two cohorts observed. FeNO predicted the gender-related differences in demographic, with women markedly over represented in the low FeNO cohort. A female preponderance in patients with chronic cough has been well documented. However, the possible relationship between different inflammatory profiles as reflected by FeNO has not previously been described.

\section{P106 THE USE OF GABAPENTIN AND PREGABALIN FOR THE MANAGEMENT OF CHRONIC COUGH IN A TERTIARY COUGH CLINIC}

${ }^{1}$ B Al-Sheklly, ${ }^{1} \mathrm{H}$ Badri, ${ }^{2}$ S Satia, ${ }^{1} \mathrm{~A}$ Woodcock, ' $\mathrm{IJA}$ Smith. 'University of Manchester, Manchester, UK; ${ }^{2}$ University Hospital South Manchester, Manchester, UK

\subsection{6/thoraxjnl-2017-210983.248}

Introduction and Objectives Chronic cough is a poorly understood condition with a limited number of treatment options available. Gabapentin and pregabalin are used in the treatment of neuropathic pain and may have some efficacy in patients with refractory chronic cough., We evaluated the real-world outcomes of using these medicines in a tertiary cough clinic.

Methods We performed a retrospective review of new referrals to a tertiary cough clinic (October 2013-October 2015). Patient characteristics (age, sex, duration of cough and test results) were collected. Follow up clinic letters were reviewed until April 2017. We recorded details regarding the prescribing of gabapentin and pregabalin for patients with refractory chronic cough, their impact on cough and the associated side effects.

Results 136 new patients were reviewed (mean age 56.3 years, $98(72.1 \%)$ female) with a mean duration of cough of 7.5 years (SD 12.2). Gabapentin or pregabalin was prescribed for 38 patients (9 gabapentin and 29 pregabalin). Highest dose achieved was $1800 \mathrm{mg} /$ day for gabapentin and $300 \mathrm{mg} /$ day for pregabalin. Overall, fifteen patients (39\%) responded favourably to these medicines initially. Fourteen (37\%) tolerated them but derived no benefit and stopped the medication. Nine patients (24\%) developed immediate side effects and were unable to tolerate the medications. Out of the 15 patients that tolerated these medicines, only 8 (21\%) were able to continue with therapy long term, as the other seven $(18 \%)$ eventually developed intolerable side effects. The most common side effect was drowsiness (see below). 